\title{
Post-thaw boar sperm motility is affected by prolonged storage of sperm in liquid nitrogen. A retrospective study
}

J unwei Li, Inmaculada Parrilla, Maria D. Ortega, Emilio A. Martinez, Heriberto Rodriguez-Martinez and J ordi Roca

The self-archived postprint version of this journal article is available at Linköping University Institutional Repository (DiVA):

http:// urn.kb.se/ resolve?urn=urn:nbn:se:liu:diva- 145795

N.B.: When citing this work, cite the original publication.

Li, J., Parrilla, I., Ortega, M. D., Martinez, E. A., Rodriguez-Martinez, H., Roca, J ., (2018), Post-thaw boar sperm motility is affected by prolonged storage of sperm in liquid nitrogen. A retrospective study, Cryobiology, 80, 119-125. https:// doi.org/ 10.1016/j.cryobiol.2017.11.004

Original publication available at:

https:// doi.org/ 10.1016/j.cryobiol.2017.11.004

Copyright: Elsevier

http://www.elsevier.com/

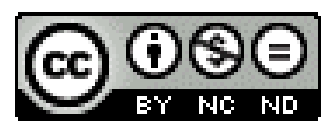


Post-thaw boar sperm motility is affected by prolonged storage of sperm in liquid nitrogen. A retrospective study.

Junwei $\mathrm{Li}^{1}$, Inmaculada Parrilla ${ }^{1}$, Maria D. Ortega ${ }^{1}$, Emilio A. Martinez ${ }^{1}$, Heriberto Rodriguez-Martinez ${ }^{2}$, Jordi Roca ${ }^{1 *}$

${ }^{1}$ Department of Medicine and Animal Surgery, Faculty of Veterinary Science, University of Murcia, Spain; ${ }^{2}$ Department of Clinical and Experimental Medicine (IKE), University of Linköping, Sweden.

*Corresponding author: Jordi Roca (roca@um.es) 


\section{Abstract}

Owing to the quick genetic turnover of the pig industry, most AI-boar sires live 2-3 yr, a period during which for 1-2 yr their semen is extended and used in liquid form for AI. Despite showing low cryosurvival, affecting fertility after AI, boar semen is frozen for easiness of transport overseas and reposition of valuable genetics. For the latter, semen is stored in liquid nitrogen ( $\mathrm{LN}_{2}$, cryostorage) for many years, a controversial practice. Here we studied how length of cryostorage could affect sperm quality. Straws $(0.5 \mathrm{~mL})$ frozen using the same cryopreservation protocol at one specific location from AI- sires of proven fertility were stored in $\mathrm{LN}_{2}$ for up to 8 yr. Post-thaw sperm quality was evaluated after 2, 4 or 8 yr of cryostorage, always compared to early thawing (15 d after freezing). Sperm motility and kinematics were evaluated post-thaw using CASA and sperm viability was cytometrically evaluated using specific fluorophores. Sperm viability was not affected by length of cryostorage, but total and progressive sperm motility were lower $(p<0.01)$ in sperm samples cryostored for 4 or 8 yr compared to those thawed $15 \mathrm{~d}$ after freezing. Cryostorage time affected sperm kinetics, but with greater intensity in the samples cryostored for 4 yr $(p<0.001)$ than in those for 2 yr $(p<0.01)$. The fact that the major phenotypic characteristic of boar spermatozoa, motility, is constrained by time of cryostorage should be considered when building cryobanks of pig semen. Attention should be placed on the finding that $>2 \mathrm{yr}$ of cryostorage time can be particularly detrimental for the post-thaw motility of some sires, which might require increasing sperm numbers for AI.

Keywords: porcine, sperm, cryopreservation, liquid nitrogen storage. 


\section{Introduction}

Freezing is the currently most efficient method for long-term preservation of mammalian spermatozoa for future use in artificial reproductive technologies [1]. Consequently, it is widely used in both humans and farm animals [2, 3, 4]. Once frozen, the spermatozoa are usually stored immersed in liquid nitrogen $\left(\mathrm{LN}_{2}\right)$. Despite early reassurances that sperm quality would not be affected $[5,6]$, concerns have been lately arisen over whether storage in $\mathrm{LN}_{2}$ (cryostorage) is totally harmless to quality and functionality of frozen-thawed (FT) spermatozoa [1, 7].

Sperm cryosensitivity differs among species [1] and porcine spermatozoa are particularly sensitive to freezing because their plasma membrane has a high proportion of polyunsaturated fatty acids but low cholesterol [8]. Accordingly, FT-boar sperm shown variable results in both functionality and fertility after thawing, leading them to be scarcely used in swine artificial insemination (AI) programs [9, 10]. This reality contrasts with the extensive worldwide use of liquid semen [11]. Despite, building cryobanks with semen from valuable boars and genetic lines for future use in AI-programs is usual practice in swine industry [10]. The putative modifications in quality or/and functionality experienced by frozen boar spermatozoa during cryostorage have received little attention. Only two studies have addressed this issue so far, whose results and conclusions might not be useful for swine $\mathrm{Al}$ because they used $6 \mathrm{ml}$ straws [12] or $10 \mathrm{ml}$ cryotubes [13] for sperm cryo-packaging, systems that are far from the one package most currently used: $0.5 \mathrm{~mL}$ plastic straws [14]. In this context, it is well known that packaging is critical for sperm cryotolerance [15]. 
The present retrospective study aimed to evaluate whether cryostorage time, up to $8 \mathrm{yr}$, could affect post-thawing attributes of boar sperm. For this purpose, cryostored sperm samples from boars with good sperm freezability, packaged in $0.5 \mathrm{~mL}$ straws and frozen and thawed using the same cryopreservation protocol at one specific location were used. Special attention was focused on kinetics, as they are sperm attributes especially sensitive to cryopreservation [16] and related with fertility post-AI [17, 18].

\section{Material and methods}

Reagents

Unless otherwise stated, all the chemicals and fluorochromes used in the experiments were of analytical grade, purchased from Sigma-Aldrich Co. (St. Louis, MO, USA). Media were prepared under sterile conditions in a laminar flow hood (MicroH; Telstar, Terrasa, Spain) using purified water (MilliQ Advantage A10, Merck, Darmstadt, Germany).

Boars, ejaculates and cryopreservation procedure

Over a period of eight years, the sperm-rich fraction of the ejaculate was collected using the gloved-hand method from healthy, mature (2-3 years old) and fertile boars of differed breeds (Landrace, Large White and Pietrain) housed in several Spanish AIcentres and regularly used as semen donors for commercial AI-programs. Immediately after collection, the ejaculates were extended (1:1, v/v) in Beltsville Thawing Solution (BTS: 205 mM glucose, 20.39 mM Na $3-\mathrm{C}_{6} \mathrm{H}_{5} \mathrm{O}_{7}, 10.0 \mathrm{mM} \mathrm{KCl,} 15.01 \mathrm{mM} \mathrm{NaHCO}$, 
3.36 mM EDTA, supplemented with 50 mg/mL kanamycin sulfate; pH 7.2 and 290-300 mOsmol/kg) and cooled to $17{ }^{\circ} \mathrm{C}$. Thereafter, they were transported overnight into insulated thermal boxes $\left(17^{\circ} \mathrm{C}\right)$ to the laboratory of Andrology of the Clinical Veterinary Teaching Hospital of the University of Murcia, where they were frozen at the morning. Only ejaculates with more than $200 \times 10^{6}$ sperm/mL, $75 \%$ of sperm motility and $85 \%$ sperm with normal morphology were used.

The ejaculates were frozen by the same working team following the standard 0.5 mL-straw protocol routinely used since 2004 [19]. Briefly, the liquid-stored semen samples were centrifuged (2,400 x g for $3 \mathrm{~min})$ and the recovered sperm pellets were reextended to $1.5 \times 10^{9}$ sperm/mL in a lactose-egg yolk (LEY) extender (80\% [v/v] $\beta$ lactose [310 $\mathrm{mM}$ in water] and 20\% [v/v] egg yolk, supplemented with kanamycin sulphate [100 $\mu \mathrm{g} / \mathrm{mL}], \mathrm{pH} 7.2$ and 295-300 mOsmol/kg). The extended samples were slowly cooled to $5{ }^{\circ} \mathrm{C}$ (during $120 \mathrm{~min}$ ) and then re-extended to $1 \times 10^{9} \mathrm{sperm} / \mathrm{mL}$ in LEY (92.5\%, v/v) plus Equex STM (1.5\%, v/v; Nova Chemical Sales Inc., Scituate, MA, USA) and glycerol (6\%, v/v;) (pH 6.2 and $1650 \pm 15 \mathrm{mOsmol} / \mathrm{kg})$. Immediately after, the samples were packed into $0.5 \mathrm{~mL}$ PVC French straws (Minitüb, Tiefenbach, Germany) and frozen in a controlled-rate freezing machine (IceCube 1810, Minitüb) using the following freezing curve: to $-5{ }^{\circ} \mathrm{C}$ at a rate of $6{ }^{\circ} \mathrm{C} / \mathrm{min}$, from $-5{ }^{\circ} \mathrm{C}$ to $-80{ }^{\circ} \mathrm{C}$ at $40{ }^{\circ} \mathrm{C} / \mathrm{min}$ rate and to $-150{ }^{\circ} \mathrm{C}$ at $70{ }^{\circ} \mathrm{C} / \mathrm{min}$ speed. The straws were plunged into $\mathrm{LN}_{2}$ and thereafter stored in large capacity LN 2 tank (GT40, Air Liquide, Paris, France) until thawing. The $\mathrm{LN}_{2}$ tank remained in a cold room $\left(5^{\circ} \mathrm{C}\right)$ and it was always operating, with regular input and output of straws. The level of $\mathrm{LN}_{2}$ within the tank continuously monitored, to ensure the straws remained always $20 \mathrm{~cm}$ below the surface of $\mathrm{LN}_{2}$.

Cryostored sperm samples 
A total of 58 cryostored sperm samples from 58 founding boars of a sperm cryobank were evaluated (one sample per boar). The cryostored sperm samples were grouped in three time-intervals of cryostorage: 2 yr (short), 4 yr (medium) and 8 yr (long), using a cryostorage time of $15 \mathrm{~d}$ as baseline control. Straws were thawed by the same working team using a standard procedure consisting in a vigorous agitation of the straws during $20 \mathrm{~s}$ inside of a circulating water bath at $37^{\circ} \mathrm{C}$. Two randomly chosen straws per cryostored sperm sample and storage time were thawed as technical replicate. Immediately after, the content of each straw was extended 1:1 (v/v) in BTS and stored in a dark chamber at $37^{\circ} \mathrm{C}$ until the completion of in vitro analyses of quality and sperm functionality.

Post-thaw sperm assessment

Sperm quality was assessed at 30 and 150 min post-thawing in terms of motility and sperm viability. Sperm motility was evaluated objectively using a computer-assisted sperm analysis system (ISAS; Proiser RtD, Paterna, Spain). The assessment procedure was that described by Cremades et al. [16]. For each evaluation, $5 \mu \mathrm{L}$ aliquots of thawed sperm samples (at $20-30 \times 10^{6} \mathrm{sperm} / \mathrm{mL}$ in BTS) were placed in a pre-warmed $\left(38^{\circ} \mathrm{C}\right.$ ) Makler counting chamber (Sefi Medical Instruments, Haifa, Israel) and 3-4 fields, with a minimum of 300 sperm per sample, were analysed. The trajectory of each identified and recorded spermatozoa was visually assessed to eliminate possible debris and to minimize the risk of including unclear tracks in the analysis. Sperm motility was recorded as the percentage of total motile spermatozoa (average path velocity $\geq 20 \mu \mathrm{m} / \mathrm{s}$ ) and the percentage of sperm showing rapid and progressive movement (straightness of the 
average path $\geq 40 \%$ ). Kinetic parameters were also recorded in FT-sperm samples cryostored during short- and medium time. Specifically, the curvilinear velocity (VCL, $\mu \mathrm{m} / \mathrm{s}$ ), straight-line velocity (VSL, $\mu \mathrm{m} / \mathrm{s}$ ), average path velocity (VAP, $\mu \mathrm{m} / \mathrm{s}$ ), linearity of sperm movement (LIN, \%), straightness of the average path (STR, \%), wobble coefficient (WOB, \%), amplitude of lateral head displacement (ALH, $\mu \mathrm{m}$ ) and beat cross frequency $(\mathrm{BCF}, \mathrm{Hz})$.

Sperm viability was evaluated in terms of plasma- and acrosome membrane integrity using a triple-fluorescence procedure with later measurement by flow cytometry (BD FACSCanto II cytometer; Becton Dickinson Co, Franklin Lakes, NJ, USA). One hundred $\mu \mathrm{l}$ of each sperm sample $\left(30 \times 10^{6}\right.$ cells/ml) was extended $(1: 9, \mathrm{v} / \mathrm{v})$ in EDTAfree phosphate-buffered saline (PBS; $139 \mathrm{mM} \mathrm{NaCl}, 2.7 \mathrm{mM} \mathrm{KCl}, 1.5 \mathrm{mM} \mathrm{KH}{ }_{2} \mathrm{PO}_{4}, 8.1$ $\mathrm{mM} \mathrm{Na} 2 \mathrm{HPO}_{4} \cdot 7 \mathrm{H}_{2} \mathrm{O}$; with $0.058 \mathrm{~g} / \mathrm{L}$ penicillin $\mathrm{G}$ and $0.05 \mathrm{~g} / \mathrm{L}$ streptomycin sulphate; pH 6.8 and 280-300 mOsmol/kg). Then, the sperm samples were stained with $3 \mu \mathrm{L}$ Hoechst 33342 (H-42; 0.05 mg/mL in PBS), $2 \mu \mathrm{L}$ propidium iodide (PI; $0.5 \mathrm{mg} / \mathrm{mL}$ in PBS) and $2 \mu \mathrm{L}$ fluorescein isothiocyanate-conjugated peanut agglutinin (PNA-FITC; 200 $\mu \mathrm{g} / \mathrm{mL}$ in PBS). Thereafter, they were incubated at $38{ }^{\circ} \mathrm{C}$ in dark for $10 \mathrm{~min}$, extended again in PBS (400 $\mu \mathrm{l})$ and then cytometrically analysed. The viable sperm population (PI negative) showing non-reacted (PNA-FITC negative) or reacted (PNA-FITC positive) acrosome were recorded.

Statistical analysis

The IBM SPSS statistics package (version 19, IBM SPSS, Madrid, Spain) was used for statistical analyses. The Shapiro-Wilk test proved that the data population of each one of the sperm parameters evaluated were non-normally distributed. Therefore, the non- 
parametric Wilcoxon signed-rank and Kruskal-Wallis one-way ANOVA tests were used to evaluate the influence of cryostorage time (15 d, as control, vs 2; 4 or 8 yr later) and the rate of decrease among the different cryostorage times, respectively, at each of the two post-thawing evaluation times (30 and $150 \mathrm{~min}$ ). $\mathrm{P}<0.05$ was considered statistically significant.

\section{Results}

Short cryostorage time (15 d, control, vs 2 yr) did not influence the percentages of total and progressive motile sperm recovered at 30 and 150 min post-thawing. However, the medium- (15 d vs 4 yr) and long ( $15 \mathrm{~d}$ vs 8 yr) cryostorage times caused a similar and significant $(\mathrm{p}<0.01)$ decrease in both variables. The decrease was similar in sperm samples evaluated at both post-thaw incubation times, and it ranged between 5 and 10\% for total motility and between 3 and 9\% for progressive motility (Figures 1 and 2).

The percentage of viable spermatozoa showing unreacted acrosomes after thawing was not affected by neither cryostorage time, irrespective of it was short, medium or long; nor by post-thaw evaluation times (Figure 3). In contrast, cryostorage duration had a negative influence on the percentage of viable spermatozoa showing reacted acrosomes, which was higher $(\mathrm{p}<0.001)$ in sperm samples cryostored during mediumand long time. However, the percentage of viable spermatozoa with reacted acrosomes was never higher than 3\%. This pattern of viable spermatozoa with reacted acrosomes was the same at both post-thaw incubation times (Figure 4).

Descriptors of kinetic parameters of motile spermatozoa recovered after thawing of sperm samples cryostored during short- and medium-time are shown in Table 1. The influence of duration of cryostorage on sperm kinetics was the same in both post-thaw 
incubation times, 30 or 150 min. Accordingly, Table 1 shows the average data of both incubation times. The kinetics of velocity, measured in terms of VCL, VSL and VAP, were negatively affected $(\mathrm{p}<0.001)$ by cryostorage time, irrespective if it was of short or medium duration. However, the influence was higher in FT-sperm samples cryostored during medium- than short-time, showing significant differences for VCL ( $\mathrm{p}<0.001)$ and VAP $(\mathrm{p}<0.05)$. Regarding the ability of spermatozoa to advance in a straight line, measured in terms of LIN, STR and WOB, the sperm cryostored for a medium-time drew more straight trajectories than those cryostored for a short-time. Accordingly, only STR increased ( $\mathrm{p}<0.001$ ) in sperm cryostored during short-time while LIN, STR and WOB increased ( $\mathrm{p}<0.001$ ) in those cryostored during medium-time. Moreover, the difference between both thawing times in the three kinetic variables was higher $(\mathrm{p}<0.05)$ in sperm cryostored for medium than for a short time. Relative to kinetics depicting sperm impulse or vigour, measured in terms of ALH and BCF, just the last one was influenced by cryostorage time, which decreased in both cryostorage times $(\mathrm{p}<0.05)$. However, the influence was higher $(\mathrm{p}<0.001)$ in samples cryostored during medium-time, as illustrated by the difference between both cryostorage periods (Table 1). Figure 5 illustrates how cryostorage time negatively affects sperm movement, making it more linear (low VCL and high LIN, Figure 5a) and less vigorous (low WOB and BCF, Figure 5b), aspects more evident in FT-sperm samples cryostored during medium- than for a short-period.

\section{Discussion}

Theoretically, cryostorage in $\mathrm{LN}_{2}$ should not impair sperm quality and functionality if sperm remain immersed in $\mathrm{LN}_{2}$, as it ensures a constant very low temperature $\left(-196{ }^{\circ} \mathrm{C}\right)$ where spermatozoa remain in a quiescent state retaining its 
functional capacities, which will be recovered at thawing [6]. Successful pregnancies achieved in humans [20, 21] and some livestock species as bovine [22] after AI with FTsperm cryostored during more than 5 years, would support the above assertion. However, the present study evidenced that FT-boar sperm lose quality during storage in $\mathrm{LN}_{2}$. The few existing studies on this subject, mainly with human semen, offer conflicting results. Some of them did not find negative effects $[7,23]$ whereas other noted a significant loss of quality and/or sperm functionality over cryostorage time [24]. Leaving out control of sperm doses during cryostorage, which should have been rigorous in all studies despite $\mathrm{NL}_{2}$ tanks remain usually operational with regular input and output of straws, the main reasons for the disagreement between the above studies would be related to semen donors, including species, individuals and even animal breeds/lines, as well as the freezing and cryo-packaging protocol used [25, 26]. The present study evaluated cryostored sperm samples from AI-breeding boars, which spermatozoa are particularly sensitive to cryopreservation [1], and the semen samples were frozen and thawed using the most currently and efficient cryopreservation protocol for boar semen, which included $0.5 \mathrm{ml}$ straws as sperm package system [14].

The present study showed that there is an apparent loss in sperm quality during cryostorage as evidenced by motility attributes, including total and progressive motility. In contrast, viability was not substantially reduced over cryostorage time. Similarly, Fraser et al. [13] using an unusual sperm packaging system for frozen boar spermatozoa, $10 \mathrm{ml}$ cryotubes, also found that cryostorage time affected motility, subjectively evaluated, rather than viability. Similarly to the study conducted by Fraser and coworkers, the present study demonstrated that the negative influence of cryostorage time on objectively evaluated total and progressive motility was not linear and progressive over time as it occurred mainly during the period between the second and fourth year of 
cryostorage without further substantial changes in subsequent years. Malik et al. [27] achieved similar results in bull sperm samples by showing a greater loss in motility in samples cryostored for 6 yr than for 2 yr. Similarly, Bolten et al. [24] evidenced that negative cryostorage effects on human FT-sperm are not progressive over time, occurring mainly within the first $3 \mathrm{yr}$ of cryostorage, being thereafter negligible. This timedependent loss in total and progressive motility of cryostored sperm samples without additional losses in viability could be explained by the findings of Desrosiers et al. [28] showing that sperm membrane damage in cryostored human semen occurs immediately after freezing whereas the damage in the cytoskeleton, specifically in $\alpha$-tubulin, can be issued during cryostorage. It is well-known that $\alpha$-tubulin is a key protein for flagellar sperm motility [29].

Currently, motile spermatozoa can be individually characterized by several kinetic parameters, which can be objectively measured using CASA systems [30]. The kinetics of thawed motile spermatozoa were also evaluated in addition to the total and progressive motile, particularly for semen frozen for 2 and $4 \mathrm{yr}$, because the substantial changes in total and progressive motility occurred between these cryostorage times. The cryostorage time also induced changes in the kinetic parameters, having especially a negative effect on kinetics for velocity and vigour. The frozen semen cryostored for $4 \mathrm{yr}$ showed at thawing a slower velocity and a lower BCF than those cryostored during 2 yr. These findings highlight that cryostorage time could impair the fertilizing ability of boar FTsemen, as kinetics are essential attributes for spermatozoa to progress in the female genital tract and to penetrate the oocytes [18]. Together, the above affected kinetic parameters, that drawing velocity and BCF, are particularly critical in porcine semen as they have been proven to be predictors of both farrowing rate [18] and litter size [17]. Moreover, their predictive value is greater in long-time preserved semen samples [31, 32], as it 
would be the case for FT-spermatozoa. The influence of sperm motility on swine fertility is especially relevant in semen AI-doses with less than $60 \%$ of motile sperm [33], which is usual in FT-semen AI-doses. Accordingly, it is not unreasonable to speculate that cryostorage beyond 2 yr can impair fertilizing capacity after thawing, owing to a decreased sperm motility together with an impairment in the kinetics of the surviving spermatozoa.

In conclusion, the present study demonstrated that cryostorage time longer to $2 \mathrm{yr}$ influenced negatively the quality of frozen-thawed boar sperm samples. The sperm attributes mainly affected were those related to motility, evidenced by a lowering in the percentages of total and progressive motile spermatozoa together with an impairment in the kinetics of the surviving thawed spermatozoa. Considering motility characteristics are the most commonly used discriminatory attributes for selecting or discarding stored semen samples, either at liquid or frozen state, for AI purposes [34]; these results should be considered when building cryobanks of boar semen because some of the cryostored semen doses may not be suitable if they remained cryostored for over 2 yr. More studies contemplating a larger number of straws and boars are needed to define the actual extent of negative effects of cryostorage time on sperm quality, which would also be focused in evaluating sperm functionality parameters and looking for putative differences among AI-boars.

\section{Acknowledgements}

The study was supported by Seneca Foundation Murcia, Spain (19892/GERM15). Junwei Li was financially supported by the China Scholarship Council. 


\section{References}

[1] J.D. Benson, E.J. Woods, E.M. Walters, J.K. Critser, The cryobiology of spermatozoa, Theriogenology 78 (2012) 1682-99.

[2] W.F. Swanson, G.M. Magarey, J.R. Herrick, Sperm cryopreservation in endangered felids: developing linkage of in situ-ex situ populations, Soc Reprod Fertil Suppl 65 (2007) 417-32.

[3] J.P. Barbas, R.D. Mascarenhas, Cryopreservation of domestic animal sperm cells, Cell Tissue Bank 10 (2009) 49-62.

[4] A. Polland, B.M. Berookhim, Fertility concerns in men with genitourinary malignancies: Treatment dilemmas, fertility options, and medicolegal considerations, Urol Oncol 34 (2016) 399-406.

[5] P.F. Watson, The preservation of semen in mammals, in: C.A. Finn (Eds.), Oxford Reviews of Reproductive Biology, Clarendon Press., Oxford, 1979, pp. 283-350.

[6] P. Mazur, Freezing of living cells: mechanisms and implications, Am J Physiol 247 (1984) C125-42.

[7] L. Yogev, S.E. Kleiman, E. Shabtai, A. Botchan, G. Paz, R. Hauser, O. Lehavi, H. Yavetz, R. Gamzu, Long-term cryostorage of sperm in a human sperm bank does not damage progressive motility concentration, Hum Reprod 25 (2010) 1097-103.

[8] S. Cerolini, A. Maldjian, F. Pizzi, T.M. Gliozzi, Changes in sperm quality and lipid composition during cryopreservation of boar semen, Reproduction 121 (2001) 395401.

[9] R.V. Knox, The Fertility of Frozen Boar Sperm When used for Artificial Insemination, Reprod Domest Anim 50 Suppl 2 (2015) 90-7. 
[10] M. Yeste, J.E. Rodriguez-Gil, S. Bonet, Artificial insemination with frozen-thawed boar sperm, Mol Reprod Dev (2017).

[11] J. Roca, I. Parrilla, A. Bolarin, E.A. Martinez, H. Rodriguez-Martinez, Will AI in pigs become more efficient? Theriogenology 86 (2016) 187-93.

[12] J. Kozumplik, Qualitative changes and the fertilizing capacity of sperm after 6 years of preservation of boar semen, Vet Med (Praha) 30 (1985) 289-300.

[13] L. Fraser, J. Strzeżek, W. Kordan, Post-thaw sperm characteristics following longterm storage of boar semen in liquid nitrogen, Anim Reprod Sci 147 (2014) 119-27.

[14] M. Yeste, Sperm cryopreservation update: Cryodamage, markers, and factors affecting the sperm freezability in pigs, Theriogenology 85 (2016) 47-64.

[15] H. Ekwall, Cryo-scanning electron microscopy discloses differences in dehydration of frozen boar semen stored in large containers, Reprod Domest Anim 44 (2009) 628.

[16] T. Cremades, J. Roca, H. Rodriguez-Martinez, T. Abaigar, J.M. Vazquez, E.A. Martinez, Kinematic changes during the cryopreservation of boar spermatozoa, J Androl 26 (2005) 610-8.

[17] C. Holt, W.V. Holt, H.D. Moore, H.C. Reed, R.M. Curnock, Objectively measured boar sperm motility parameters correlate with the outcomes of on-farm inseminations: results of two fertility trials, J Androl 18 (1997) 312-23.

[18] M.L. Broekhuijse, E. Sostaric, H. Feitsma, B.M. Gadella, Application of computerassisted semen analysis to explain variations in pig fertility, J Anim Sci 90 (2012) 779-89.

[19] G. Carvajal, C. Cuello, M. Ruiz, J.M. Vázquez, E.A. Martínez, J. Roca, Effects of centrifugation before freezing on boar sperm cryosurvival, J Androl 25 (2004) 38996. 
[20] S. Kelleher, S.M. Wishart, P.Y. Liu, L. Turner, I. Di Pierro, A.J. Conway, D.J. Handelsman, Long-term outcomes of elective human sperm cryostorage, Hum Reprod 16 (2001) 2632-9.

[21] J. Feldschuh, J. Brassel, N. Durso, A. Levine, Successful sperm storage for 28 years, Fertil Steril 84 (2005) 1017.

[22] T. Haugan, Y.T. Gröhn, E. Kommisrud, E. Ropstad, O. Reksen, Effects of sperm concentration at semen collection and storage period of frozen semen on dairy cow conception, Anim Reprod Sci 97 (2007) 1-11.

[23] A. Ramírez-Reveco, J.L. Hernández, P. Aros, Long-Term Storing of Frozen Semen at $-196^{\circ} \mathrm{C}$ does not Affect the Post-Thaw Sperm Quality of Bull Semen, in: F. Marco-Jiménez, H. Akdemir, (Eds.), Cryopreservation in Eukaryotes, InTech, Rijeka, 2016, pp. Ch. 06.

[24] M. Bolten, L. Weissbach, R. Kaden, Cryopreserved human sperm deposits: usability after decades of storage, Urologe A 44 (2005) 904-8.

[25] E.J. Woods, J.D. Benson, Y. Agca, J.K. Critser, Fundamental cryobiology of reproductive cells and tissues, Cryobiology 48 (2004) 146-56.

[26] H. Ekwall, M. Hernandez, F. Saravia, H. Rodriguez-Martinez, Cryo-scanning electron microscopy (Cryo-SEM) of boar semen frozen in medium-straws and MiniFlatPacks, Theriogenology 67 (2007) 1463-72.

[27] A. Malik, M. Laily, M.I. Zakir, Effects of long term storage of semen in liquid nitrogen on the viability, motility and abnormality of frozen thawed Frisian Holstein bull spermatozoa, Asian Pac J Repro 4 (2015) 22-25.

[28] P. Desrosiers, C. Légaré, P. Leclerc, R. Sullivan, Membranous and structural damage that occur during cryopreservation of human sperm may be time-related events, Fertil Steril 85 (2006) 1744-52. 
[29] C. Gagnon, D. White, J. Cosson, P. Huitorel, B. Edde, E. Desbruyeres, L. PaturleLafanechere, L. Multigner, D. Job, C. Cibert, The polyglutamylated lateral chain of alpha-tubulin plays a key role in flagellar motility, J Cell Sci 109 ( Pt 6) (1996) 154553.

[30] R.P. Amann, D. Waberski, Computer-assisted sperm analysis (CASA): capabilities and potential developments, Theriogenology 81 (2014) 5-17.e1-3.

[31] N. Sutkeviciene, M.A. Andersson, H. Zilinskas, M. Andersson, Assessment of boar semen quality in relation to fertility with special reference to methanol stress, Theriogenology 63 (2005) 739-47.

[32] M. Schulze, K. Ruediger, K. Mueller, M. Jung, C. Well, M. Reissmann, Development of an in vitro index to characterize fertilizing capacity of boar ejaculates, Anim Reprod Sci 140 (2013) 70-6.

[33] W.L. Flowers, Management of boars for efficient semen production. J Reprod Fertil Suppl 52 (1997) 67-78.

[34] P. Kathiravan, J. Kalatharan, G. Karthikeya, K. Rengarajan, G. Kadirvel, Objective sperm motion analysis to assess dairy bull fertility using computer-aided system--a review, Reprod Domest Anim 46 (2011) 165-72. 


\section{Figure legends}

Figure 1. Total sperm motility of frozen boar sperm samples stored in liquid nitrogen during short ( 2 yr), medium ( 4 yr) or long ( 8 yr) times after freezing, always compared to early thawing (15 d) for each respective semen sample. Whisker-box plots on the left (white bars) show the raw percentages of two assessments after thawing (at 30 and 150 min of incubation at $37^{\circ} \mathrm{C}$ ) recorded in cryostored sperm samples thawed at two different times after freezing: $15 \mathrm{~d}$ (control) vs 2, 4 or 8 yr later. Histograms (mean \pm SEM) on the right (black bars) show the difference in the percentage of total motile sperm between the two cryostorage times (15 d, control, vs short, medium or long time). Whisker-box shows the 25th and 75th percentiles (box), the 5th and 95th percentiles (whisker) and median (line within box). $\mathrm{m}, \mathrm{n}$ and $\mathrm{x}$, $\mathrm{y}$ indicates differences $\mathrm{p}<0.01$ and $\mathrm{p}<0.001$, respectively.

Figure 2. Progressive sperm motility of frozen boar sperm samples stored in liquid nitrogen during short ( 2 yr), medium ( 4 yr) or long ( 8 yr) times after freezing, always compared to early thawing (15 d) for each respective semen sample. Whisker-box plots on the left (white bars) show the raw percentages of two assessments after thawing (at 30 and 150 min of incubation at $37^{\circ} \mathrm{C}$ ) recorded in cryostored sperm samples thawed at two different times after freezing: $15 \mathrm{~d}$ (control) vs 2, 4 or 8 yr later. Histograms (mean \pm SEM) on the right (black bars) show the difference in the percentage of progressive sperm motility between the two cryostorage times (15 d, control, vs short, medium or long time). Whisker-box shows the 25th and 75th percentiles (box), the 5th and 95th 
percentiles (whisker) and median (line within box). a, b; m, n and $\mathrm{x}, \mathrm{y}$ indicates differences $\mathrm{p}<0.05, \mathrm{p}<0.01$ and $\mathrm{p}<0.001$, respectively.

Figure 3. Sperm viability of frozen boar sperm samples stored in liquid nitrogen during short ( 2 yr), medium (4 yr) or long (8 yr) times after freezing, always compared to early thawing (15 d) for each respective semen sample. Whisker-box plots on the left (white bars) show the raw percentages of two assessments after thawing (at 30 and 150 min of incubation at $37^{\circ} \mathrm{C}$ ) recorded in cryostored sperm samples thawed at two different times after freezing: $15 \mathrm{~d}$ (control) vs 2, 4 or 8 yr later. Histograms (mean \pm SEM) on the right (black bars) show the difference in the percentage of viable sperm between the two cryostorage times (15 $\mathrm{d}$ and short, medium or long time). Whisker-box shows the 25th and 75th percentiles (box), the 5th and 95th percentiles (whisker) and median (line within box).

Figure 4. Viable spermatozoa with reacted acrosome of frozen boar sperm samples stored in liquid nitrogen during short ( 2 yr), medium ( 4 yr) or long ( 8 yr) times after freezing, always compared to early thawing (15 d) for each respective semen sample. Whisker-box plots on the left (white bars) show the averaged raw percentages of two assessments after thawing (at 30 and 150 min of incubation at $37^{\circ} \mathrm{C}$ ) recorded in cryostored sperm samples thawed at two different times after freezing: $15 \mathrm{~d}$ (control) vs 2, 4 or 8 yr later. Histograms (mean \pm SEM) on the right (black bars) show the difference in the percentage of viable sperm with reacted acrosome between the two cryostorage times (15 d and short, medium or long time). Whisker-box shows the 25th and 75th percentiles (box), the 5th and 95th percentiles (whisker) and median (line within box). m$\mathrm{o}$ and $\mathrm{x}, \mathrm{y}$ indicates differences $\mathrm{p}<0.01$ and $\mathrm{p}<0.001$, respectively. 


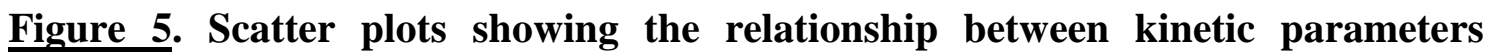
recorded after thawing in frozen-thawed boar sperm samples stored in liquid nitrogen during short ( 2 yr) or medium (4 yr) time after freezing, always compared to early thawing (15 d) for each respective semen sample. The figures on the left (A) show the relationship between velocity (curvilinear velocity, VCL) and linearity (LIN) and those on the right (B) the relationship between linearity (degree of oscillation of the actual sperm-head trajectory around its average path, WOB) and vigor (beat cross frequency, BCF). The number 1 represents data recorded in sperm samples cryostored during $15 \mathrm{~d}$ (control, grey) or $2 \mathrm{yr}$ (black) at the number 2 data recorded in sperm samples cryostored during $15 \mathrm{~d}$ (control, grey) or 4 yr (black). 